\title{
Towards a Causal Framework for Intelligent Agents Development
}

\author{
Hector G. Ceballos and Francisco J. Cantu \\ Center for Intelligent Computing and Robotics \\ Tecnologico de Monterrey, Campus Monterrey \\ $\{$ ceballos,fcantu\}@itesm.mx
}

\begin{abstract}
In this paper we present a Causal Artificial Intelligence Design (CAID) theory that borrows notions from Classical philosophy for modeling intelligent agents. Principles introduced by this theory are used for extending a goal-driven BDI architecture and implementing what we call Causal Agent. This architecture incorporates causal formalisms like Pearl's Do calculus and $\mathrm{C}+$ which are adapted to Semantic Web knowledge representations. Our approach includes an ontological agent description that enables and justifies the instantiation of agents as part of a plan. An experimental prototype used for validating experimentally our approach is commented.
\end{abstract}

\section{INTRODUCTION}

Development of intelligent agents have originated several architectures and approaches ranging in complexity from the reactive to the rational agent. The use of mental states and explicit intentions have produced one of the most accepted approaches [9]. Nevertheless, as long as the complexity of domains increases, general assumptions become insufficient to tackle every problem. In SOAR [12], Newell incorporates semantic knowledge in order to formalize common sense. In LORA [17], Wooldridge introduces a first order component to represent the properties of objects within the application domain.

The assumption of self-interested agents with unknown purposes makes a really hard task to determine if a coalition with other agent will be profitable for both parts. For this purpose, cooperation between agents have been modeled through shared intentions [7] or social commitments [16], among others. Encoding utilitarian theories in agents' reasoning have proved to be an efficient mechanism for agent's decision but finally requires knowledge about all the participants when a global goal must be achieved [15]. All these approaches have an individual perspective where an agent is considered intelligent as long as can achieve its goals, and where cooperation is justified as long as reports individual benefits.

We introduce an approach for modeling intelligent agents from a causal perspective borrowed from classical philosophy. Causality and Metaphysics provide a coherent framework for delimiting the capabilities and rationality of an agent and for modeling processes in terms of involved entities and cause-effect relationships. Recent mathematical formalisms supporting at a given extent both theories are applied.

This paper is organized as follows: in section II are presented some philosophical foundations of our approach; section III presents rector principles of our proposal, meanwhile section IV introduces an agent architecture based on these principles, including an actual implementation. In section $\mathrm{V}$ is compared our approach with other existing ones and in section VI are given some closing remarks.

\section{Philosophical Foundations}

Aristotle unified classic realism and idealism in a middle position that proposed that real things are the material representation of forms called essences, abstract ideas which are materialized in concrete entities. This idea has evolved through the history of mankind, discussing mainly the real existence and precedence of matter and forms.

Aristotle conceived reality constituted by entities or beings which common characteristics are abstracted by human mind in concepts named essences [1]. These characteristics or accidents were classified by Aristotle as intrinsic, extrinsic and mixed. Intrinsic accidents include quantitative (age, size, etc.), qualitative (color, shape, etc.) and relational (fatherhood, nationality, etc.) accidents, that is, what internally identify an entity. Extrinsic accidents are relative to time (birth date, duration, etc.), place (position), possession (property) and disposition (sit, stand, etc.). Mixed accidents explain interaction among entities: action is present in an entity when originates movement or change in another, meanwhile affection is present in entities that receive passively the action of another.

Aristotle used the notion potency for representing the capacity of an entity for showing certain accident. Act, on the other hand, is the actual presence of the accident on the entity. Having certain accident in potency doesn't imply that the entity shows it actually, but only denotes possibility. For example, a given statue can be described by its actual accidents (size, color, antiquity, location, creator, etc.). On the other hand, an sculptor is a person that in potency can sculpt an statue and receive a payment for it.

Aristotle contributed to the contemporaneous causality theory too. Causality refers to the set of all particular "causal" or "cause-effect" relations. Most generally, causation is a relationship that holds between events, properties, variables, or states of affairs. Causality implies at least some relationship of dependency between the cause and the effect. Cause chronologically precedes the effect. All possible causes fall into four main groups. The material cause refers to the matter or the substratum. The formal cause identifies the essence, the pattern, the form, or the structure. The efficient cause 
is the primary moving change or the agent and its action (agent causation). Finally, the final cause is the goal or the end. According to this theory causes that intervene in any change, including the creation of entities, falls in one of these categories. The entity that receives the effect of this change is called caused thing.

Brentano defines intentionality as a characteristic of "mental phenomena", by which they could be distinguished from "physical phenomena". This definition of mental phenomena is used for distinguishing objects from agents. Meanwhile both are defined as entities, the former lacks of mental states, and hence intentionality.

Continuing with our example, we can identify the main causes of the Caesar's statue at Louvre: its formal cause is Julius Caesar, its material cause is the rock of which it was made, its efficient cause is the sculptor Nicolas Coustou, and its final cause would had been to receive some payment for it. The rock received passively the action of Coustou who was who had the intention of making the statue from it.

\section{A. Modern Computational Models}

The recent development of computer science have provided mathematical models that support both theories at a given extent. On one side we have the probabilistic formalism proposed by Judea Pearl [13] that allows identifying causal relations through the observation of a phenomenon (or process) and quantifying the causal effect of the intervention of a random variable over another (acting). Besides, Pearl proposes a method for detecting unobserved causes that explain the unpredictable outcome of an observed phenomenon (contingency). On a similar way, the nonmonotonic causal logic $\mathrm{C}+$ [10] is the first formal language the expresses cause-effect relations and makes temporal precedence considerations.

On the other hand, Description Logics [3] have extended first order logic with constructors that expresses relations and constraints among concepts enabling to express domain dependent ontologies. Developed inference mechanisms allows to check the consistency of definitions and to generate possible worlds according to the given set of definitions and assertions. DL conjunctive queries allows to express patterns on which containment is decidable [11]. An action formalism based on DL has been proposed [2].

\section{Causal ARtificial Intelligence Design}

We present the Causal Artificial Intelligence Design (CAID) approach, constituted by a set of principles that guide the design of intelligent agents and Multi-Agent Systems:

1) Total Causality [TC]. Any software agent or system can be specified and instantiated through its four main causes: formal, material, efficient and final.

2) Intentional Causation [IC]. Every agent of a system is created with a purpose (final cause) derived from an upper level plan.

3) Ontological Commitment [OC]. Agents will only commit to achieve goals consistent with its essence (formal cause) and will act in consequence.
4) Causal Effect Accountability [CEA]. Agent decisions are based on the accountable causal effects of its actions.

According to the Total Causality principle, an agent can be instantiated through its main causes. The formal cause answers to the question how is it? with an agent class defined in terms of accidents or properties the agent can and must show, i.e. its potential and essential properties. The efficient cause answers the question who creates it? with the identifier of the agent that instantiate it. The material cause answers the question what does compose it? with the software implementations for the agent itself and its components (sensors and actuators). The final cause answers the question why is it created? and it's given in terms of those goals that the agent must achieve in the instantiator's plan. Even when the final cause is not a parameter on the agent's instantiation, it's the justification of its instantiation.

The agent class include the type of actions it can perform and the kind of goals it can achieve. If the capabilities of agent classes are known and their instantiation is controllable, planning can consider actions and plans controlled by non-existing agents. The resulting plan must indicate the sequence of actions required for achieving the original goal, as well as the set of agents that will perform them, given by tuples PlanOperation(operation , $_{i}$ agent $_{i}$ ). For a tuple PlanOperation $\left(o p_{1}, a g_{1}\right)$ where $a g_{1}$ doesn't currently exist, the instantiation of $a g_{1}$ is enabled by the Total Causality principle. The Intentional Creation principle proposes that the instantiation of $a g_{1}$ has $o p_{1}$ as final cause. If the created agent has the capability of instantiating other agents, this instantiation can be done recursively. The original goal, owned by the former agent, is decomposed hierarchically through this process.

The kind of goals the agent must achieve according to its ontological definition (agent class) are called essential goals. The Ontological Commitment principle establishes that ontologically, i.e. in order to be consistent with its definition, an instance of this agent class can only agree to achieve goals compatible with its essential goals. The internal state of the agent will determine if the agent can or cannot accept such goal. Once that the agent commits to achieve a new goal it will execute all the available plans until achieving the goal (success) or exhaust them (fail). In both cases, the agent delegating the goal must be informed of the outcome.

The Causal Effect Accountability principle establishes that an agent needs to know the effects of its own actions in order to choose those that will allow him to achieve the goals it has committed to. Using Pearl's notation [13], the causal effect of action $a$ over goal $g$ given current conditions $c$ is given by $P(g \mid d o(a), c)$. As long as this effect is identifiable, i.e. exists a causal model with unbiased causal paths from $a$ to $g$ and there is enough evidence in $c$, it can be determined how effective will be performing $a$ w.r.t. $g$.

If the agent can identify or is informed of indirect effects of its actions it can consider them to make better decisions. Additionally, the creator agent can inform to the created agent how desirable (or not) are such effects, enforcing (or 
inhibiting) agents actions. This last attribution will allow to indicate the agent "how well or bad is it doing it". Causal relations and preferences are delivered by the agent responsible for a plan to those agents that execute it.

\section{Causal Agent}

We introduce a design methodology and agent architecture that uses the principles of CAID for development of intelligent agents. The proposed architecture extends the goal-driven BDI architecture with a set of elements and operations. An implementation with existing formalisms is proposed at the end of this section.

Definition A Causal Agent is an intentional entity modeled [TC] and instantiated [IC] through the four main causes that is considered intelligent as long as it achieves goals consistent with its definition [OC] and makes decisions based on the causal effects of its actions [CEA].

\section{A. CAID architectural elements}

The agent class is defined using Description Logics formalisms [3] (see Figure 1) that support current Semantic Web Ontology language OWL ${ }^{1}$. This definition is published allowing other agents to inspect the capabilities of such agents and enabling the instantiation of agents with the minimum requirements indicated on it. The agent class contains: potential and essential properties, goals it can achieve, rules that modify its internal state, controllable plans and components used for sense and act on the environment. Nevertheless, exposing properties and goals would be enough for determining if the agent can participate or not in a plan.

\begin{tabular}{|l|l|}
\hline Constructor & Syntax \\
\hline Top & $\top$ \\
Bottom & $\perp$ \\
Concept inclusion & $C_{1} \sqsubseteq C_{2}$ \\
Role inclusion & $R_{1} \sqsubseteq R_{2}$ \\
Intersection & $C_{1} \sqcap \ldots \sqcap C_{n}$ \\
Value restriction & $\forall R . C$ \\
Qualified exact restriction & $=n R . C$ \\
Qualified at-least restriction & $\geqslant 0 R . C$ \\
Qualified at-most restriction & $\leqslant 0 R . C$ \\
\hline
\end{tabular}

Fig. 1. DL constructors used on agent definition.

1) Agent Class.: The agent class is represented by a concept name and its definition is given by a set of constraints that identify the roles (accidents) that can be associated to the agent. The agent class has the general form:

$$
\text { AgClass } \sqsubseteq \text { SupCls } \sqcap \geqslant 0 R_{i} . C_{i} \sqcap=n R_{j} . C_{j} \sqcap R_{k}=V_{k}
$$

for $0 \leq\{i, j, k\} \leq m$, where AgClass is the name of the agent class, $S u p C l s$ is the agent class from which inherits properties and constraints, $R_{i}$ are potential properties with

\footnotetext{
${ }^{1}$ World Wide Web Consortium (W3C). Web Ontology Language (OWL). http://www.w3.org/2004/OWL/
}

range on objects of the class $C_{i}, R_{j}$ requires $n$ instances of type $C_{j}$, and $R_{k}$ is set to $V_{k}$ by default ( $V_{k}$ can be a known instance or a literal value). Constraints expressed through $R_{j}$ and $R_{k}$ are considered essential properties that any instance of $A g C l a s s$ must satisfy.

This definition of agents allows to express a specialized hierarchy of agents. Concepts SoftAg and HumanAg, descendants of Agent are used for representing the class of software agents and human agents. Human $\mathrm{Ag}$ subclasses denote roles played by humans in the system. The role type (accident category) can be indicated through a role hierarchy and the use of constant roles like $R_{\text {action. }}$. If the agent can perform action $\alpha$ then role $R_{\alpha}$ is included as potential role on the agent's definition and it is asserted the relation $R_{\alpha} \sqsubseteq R_{\text {action }}$ in the schemas definition (TBox).

2) Essential goals.: Essential goals are represented in the definition with the constructor AgClass hasEssentialGoal $=G$ where $G$ is of type Goal. A goal has the general form Goal(pre, final, cancel, until) where pre is the condition that describes the entities required in terms of actual properties, final is another condition indicating the desired state of such entities in terms of potential properties, cancel is the cancelation condition (ending with failure), until is the termination condition (ending with success). All the conditions are represented by conjunctive queries, over which query containment can be determined $\left(Q_{1} \sqsubseteq Q_{2}\right)$ [11]. The use of until $=\perp$ indicates an indefinite goal, on which achieving final doesn't imply the termination of the goal.

3) Rules.: Rules are associated with the constructor knows Rule $=R$ where $R$ is of type Rule. Rules are used for transforming perceptions into beliefs and for revising beliefs. Rules can be used too for describing the possible outcomes of an action execution. Rules' predicates include concepts and roles names used for defining the agent and other objects in the domain.

4) Plans.: Plans are described semantically indicating: required internal conditions ( $r e q$ ), the triggering condition (pre), the final condition ( inal) and the sequence of actions to execute. Depending on the actions contained in the plan and the capabilities of an agent it is possible to determine if the agent can execute the entire plan (total control) or just part of it (partial control), i.e. without or with assistance of other agents. This condition can be codified through role assertions of the type controlsPlanT(AgClass, Plan) or controlsPlanP(AgClass, Plan), respectively. Even when this calculation can be expensive computationally, it can be done off line. Satisfiability of goal $G$ by plan $P$ can be calculated evaluating G.pre $\sqsubseteq$ P.pre $\wedge$ G.final $\sqsubseteq$ P.final, where P.pre and P.final are logical formulas expressed as conjunctive queries that represent the initial and final state associated to the plan execution. Goal satisfiability is persisted through the role assertion satisfyGoal $(P, G)$.

5) Components.: Sensors and actuators are implemented in Java through given interfaces. These interfaces allow sensors to gather information and store it on perceptions, and actuators to execute actions indicated in the beliefs set. Both 
of them are considered agent components and are described semantically as concepts (classes) that are associated to the agent class through the constraint $=n$ hasSensor. $S_{1}$ (or $=n$ has Actuator. $A_{1}$ ), indicating the number of sensors (or actuators) of type $S_{1}$ (or $A_{1}$ ) the agent must posses. Component description must include references to its software implementation and a list of affection or action roles that implement. For example, constraint $A_{1} \sqsubseteq$ implements $=R_{\alpha}$, indicates that actuator $A_{1}$ implements the action role $R_{\alpha}$. On a similar way, the Java implementation of the agent is indicated through a similar constraint, AgClass $\sqsubseteq$ hasImplementation = Impl, where Impl describes the Java class and the code location.

\section{B. CAID Functions}

Additionally to functions performed in a goal-driven BDI agent, CAID principles introduce new functionality.

1) Agent instantiation.: Denoted by an action role, described by an action rule and implemented through an actuator, agent instantiation is achieved indicating: the agent class, the instantiator agent (ID and type), and the identifier for the new agent. The agents platform must provide the appropriate functionality. During set up, the agent implementation load instances of sensors and actuators implementations, set default values and loads essential goals, controlled plans and known rules, all of them indicated on its definition. Finally, it associates the constant Self used on rules and plans, to its assigned identifier. If some exception is raised during this process, it notifies to its efficient cause; otherwise notifies the successful instantiation.

2) Intention transmission.: The operation $O p_{1}$ that motivated the instantiation of agent $A g_{1}$ is transmitted to the instantiated agent as a goal through a commitment. The commitment has the form $X C C(d b, c r, G, w)$, where $d b$ is the debtor agent, $\mathrm{cr}$ is the creditor agent, $G$ is the goal to achieve, and $w$ is the importance factor assigned by $\mathrm{cr}$ in relation to the upper level goal from which $G$ was derived. If $G$ is contained in some agent's essential goal $G_{0}$, denoted $G \sqsubseteq G_{0}$, and the internal conditions ( $r e q$ ) for at least one plan satisfying $G$ are set, the commitment is accepted; otherwise, it is rejected.

Goal containment is done by evaluating G.pre $\sqsubseteq G_{0}$.pre $\wedge$ $G$. final $\sqsubseteq G_{0}$. final. Once that the agent accepts a commitment for this goal, it activates those plans that satisfy the respective $G_{0}$. Due to query containment, satisfyGoal $\left(P, G_{0}\right)$ and $G \sqsubseteq G_{0}$ implies that $\operatorname{satisfy} \operatorname{Goal}(P, G)$.

3) Planning.: Planning is an expensive task that only certain agents must be enabled to do. Implemented through an actuator, planning receives a goal as input and can be done on two ways: considering agent instantiation as an operator in a logic program, or using an algorithm that apply essential goals as operators and latter determines which agent would be the best candidate for it (instantiating it if there is none available).

In both cases, the plan produced by this algorithm must throw: a set of agents to instantiate and a set of commitments to delegate. Planning can produce more than one feasible plan due to: multiple paths for reaching the goal, goals in common among different agent classes, and the possibility of delegating the commitment to an existing or a new agent. From the set of feasible plans, one must be selected. Plan selection can use heuristics like minimizing the number of commitments or maximizing the probability of success of the sequence of actions.

4) Plan deployment.: Plan deployment consists on instantiating required agents and delegating commitments in the plan. If some agent doesn't accept one of the commitments in the plan, the commitment can be assigned to another of the same class or to a new agent. Once that the goal is achieved, commitments derived from the plan are released and agents without active commitments are disposed.

5) Commitment operations.: The following functionality of Singh's commitment formalism is incorporated: 1) definite commitments are discharged by the agent once that the until condition is met, 2) final's condition achievement of indefinite commitments is notified to the creditor, 3) cancelation of a commitment is notified once that the cancel condition holds, 4) a commitment can be discharged by the creditor. Discharging, releasing or cancelation of commitments motivates the revision of goals and active plans.

6) Causal relations exchange.: Causal relations are exchanged using standard communication protocols in the format $C R$ (cause, effect, likelihood) where cause and effect are conditions represented by conjunctive queries, and likelihood represents the conditional probability $P($ effect $\mid$ cause $)$ that the sender beliefs. This information can be used for extending the causal network of an agent or can be considered for adjusting the probability of an existing causal relation.

Additionally, preferences can be modeled associating a cost or utility $C$ to certain condition $P$, denoted Preference $(P, C)$ on which $C$ is positive for indicating preference and negative for indicating an undesired condition. The absolute value of $C$ indicates how preferred or not is $P$. Preferences are searched over the nodes of a plan in order to include the cost $C$ in the action selection procedure.

\section{Implementation}

The implementation of a Causal Agent extends a goal-driven BDI architecture adding the elements illustrated in Figure 2 and redefining some of the actual functions of the original approach. Our current implementation is made using the JADE framework [4]. The domain ontology, that includes agents and objects, as well as the core ontology containing the elements described above are defined using OWL. The Jena Framework ${ }^{2}$ is used for loading the domain ontology, maintaining sets of assertions (ABoxes) in memory, and applying semantic reasoning derived from the definitions and the given entailment rules. Currently, it is applied only RDFS reasoning, i.e. object oriented reasoning, which is computed in polynomial time.

\footnotetext{
${ }^{2}$ Jena - A Semantic Web Framework for Java. http://jena.sourceforge.net/
} 


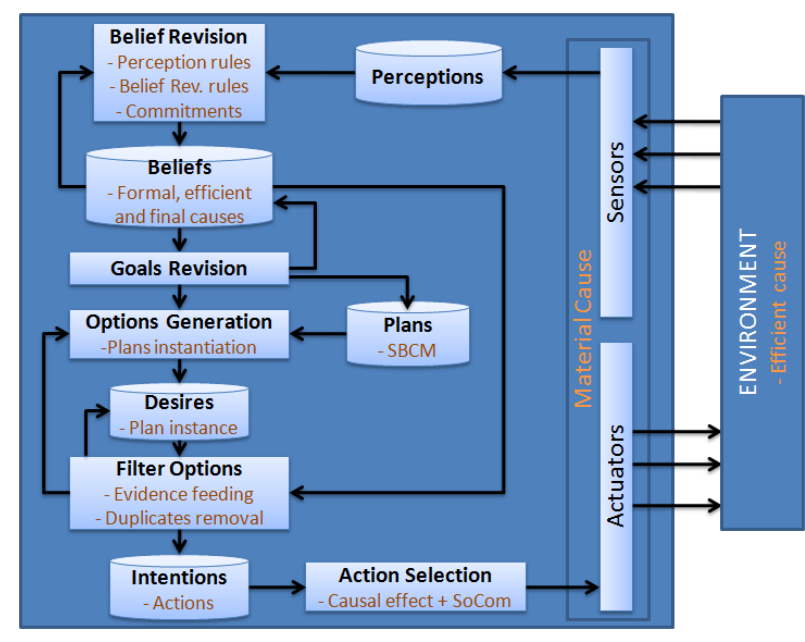

Fig. 2. CAID BDI architecture.

1) Java Interfaces.: The Java Sensor interface requires the implementation of the function boolean sense ( $A B O x$ perceptions) which stores the information gathered in the ABox perceptions using the schemas defined in the domain ontology, and returns True if something is perceived. The perception must be consistent with the sensor description $S$ that establishes implements $\left(S, R_{\rho}\right)$, where the affection role $R_{\rho}$ has for range the type of objects perceived; for instance, an ACL message. Similarly, the Java Actuator interface implements the function boolean act ( $A B O x$ beliefs, ABox nextBeliefs) which looks in the beliefs Abox for instances of the action roles it implements in order to trigger their execution. The result of its execution is updated in the nextBeliefs ABox and the function returns True if at least one action was executed.

2) ABoxes.: Beliefs and perceptions are represented by independent ABoxes. Along the inference process there are two versions of the beliefs set: the previous beliefs set and the current beliefs set. Both beliefs set are almost the same, except that the former stores the actions executed in the last step and the latter stores the effects of such actions. In the beliefs set can be found the agent definition, its efficient cause and its active goals.

3) Rules.: Belief revision rules are implemented as RDF rules and executed with the Jena inference support on the current set of beliefs. For the incorporation of perceptions into beliefs is used a rule representation with the form PercRule (perc, curr, $\left.e^{+}, e^{-}\right)$, where perc and curr are conjunctive queries executed over the current set of perceptions and beliefs, respectively; results obtained from perc are replaced in curr and the last result is used for constructing statements from $e^{+}$and $e^{-}$that are added or removed in the current set of beliefs. The execution of these rules is made through SPARQL select query executions. The rule engine has the characteristic of creating instances on runtime for representing new individuals added by $e^{+}$.

4) Goals Revision.: After beliefs revision is performed a procedural checking of goals' satisfaction and commitments' discharging. final and until goals' conditions are evaluated as SPARQL select queries in the updated set of beliefs. Commitments discharges and goals achievements are prepared as messages to the respective creditors. Active goals and plans are revised.

5) Plans.: Plans are represented by a formalism that integrates Bayesian networks and semantic annotations [6]. Annotations, represented by DL conjunctive queries, allows to identify action roles in a node and based on the agent class capabilities, controllability of the plan can be determined. The execution order is given by the causal structure and goals achievable by the plan are given by the annotations of the final nodes $(F)$. This formalism was complemented with a set of realizations $\left(Z_{i}=z_{i}\right)$ identifying the context on which the plan can be triggered; annotations of these realizations are used for identifying the precondition in terms of queries that can be executed over the beliefs set.

6) Options.: Options generation consists on identifying those active plans that can be instantiated from the current beliefs set. Annotations of SBCM contexts are evaluated as SPARQL select queries on the current set of beliefs. For every tuple returned by this evaluation a new SBCM instance is created. A SBCM instance is represented by $S B C M \operatorname{Inst}(B, S)$ where $B=\left\{\left(V_{i}=v_{i}\right) \mid i>0\right\}$ is a set of realizations of the Bayesian network and $S=\left\{\left(S_{i}=s_{i}\right) \mid i>0\right\}$ is a set of realizations for semantic variables produced by the evaluation of annotations on $V$.

During goals revision, options with pending nodes are evaluated in the current set of beliefs. Options completely revised are used for parametric learning of the model and removed from the set of current options. Options filtering is used for discarding duplicated options. A new option is considered duplicated if all the semantic variables of its context are contained in the set of semantic variables of a current option.

7) Intentions.: Intentions are those actions that can be executed from the current set of options. An executable action is represented by a controllable node $X$ not intervened and which preconditions are set, i.e. $P(X=$ True $\mid E=e) \geq \tau$, where $E=e$ is the evidence stored in the option and $\tau$ is a threshold that indicates under how much uncertainty the agent can act, usually in the range $[0.8,1.0]$.

Action selection considers the causal effect of all feasible actions over the final state of the plan and over the preferences identified on it. The causal effect of the action $X$ over the final state of the plan $F=f$ is calculated by $\operatorname{ce}(F)=$ $P(F=f \mid d o(X=$ True $), E=e)$. A preference $\operatorname{Pre}_{1}=$ $\operatorname{Pref}\left(P_{1}, C_{1}\right)$ is identified in the plan if exist some observable variable $Z_{p}$ in the plan such that $P_{1} \sqsubseteq \operatorname{Ann}\left(Z_{p}=z_{p}\right)$. The causal effect over such preferences is calculated on a similar way, $\operatorname{ce}\left(\operatorname{Pref}_{1}\right)=P\left(Z_{p}=z_{p} \mid \operatorname{do}(X=\right.$ True $\left.), E=e\right)$. On this way, the score of action $X$ is calculated by:

$$
\operatorname{score}(X)=c e(F)+\sum_{i} c e\left(\operatorname{Pref}_{i}\right) \cdot C_{i}
$$

for all preferences Pref $f_{i}=\operatorname{Pref}\left(P_{i}, C_{i}\right)$ identified in the plan. If $\operatorname{score}(X)<\tau$ the action is inhibited. 
The action $X$ with the highest score is selected and its annotation $\operatorname{Ann}(X)$ is used for encoding the action to execute. Action encoding consist on replacing constants from the SBCM instance in $\operatorname{Ann}(X)$ and insert the resulting statements in the beliefs ABox.

\section{DisCUSSION}

Unlike methodologies like Gaia that require an additional processing of its specification, our proposal uses the ontology formalism for documenting the domain and designing agents on an expressive formal language.

Potential accidents included in the definition of an agent allows to identify those objects that can be found in the agent's beliefs set and that can be accessed by rules and actuators; such definition becomes cumbersome in Jadex. Agent's definitions and the formalization of the instantiation act allows that agents can consider the inclusion of a non-existing agent in planning. The framework allows to perform a hierarchical decomposition of a task allowing an agent to delegate subtasks or subgoals to other agents. As a consequence, it allows to establish protocols dynamically through commitments delegation. Hierarchical planning like the one performed by SOAR [12] requires a global perspective, which we achieve indicating in the essential goals only those aspects of the plan that affect external objects putting aside implementation details.

Inference is made at three levels: semantic, logic and probabilistic. The use of ABoxes for representing beliefs allows to introduce RDFS entailment. Belief revision and perceptions incorporation is controlled through rules compatible with ABoxes. Finally, the SBCM approach allows to close the bridge between the ABox and a probabilistic representation.

The probabilistic representation of plans in Bayesian Network allows to calculate indirect effects beyond a series of actions, something that $\mathrm{C}+[10]$ can only do on a scope of a time step. Agents can calculate the tradeoff between individual intentions and global preferences, inhibiting or enforcing some actions. Similar work has been done introducing custom factors in rules used by agents [5].

Unlike Jadex [14], our approach provides a plan representation that allows synchronizing plan execution through action selection and transfers Java implementation to the layer of sensors and actuators. SBCM instances are used for performing Bayesian parametric learning on the model, which allows to modify the behavior of the agent or detect ineffective plans.

\section{CONClusions}

We presented the Causal Artificial Intelligence Design, a theory for modeling intelligent agents based on philosophical causality theory. Aristotelian metaphysics and causality theory is used for defining intelligent agents. We introduced the Causal Agent architecture based on this theory and documented an implementation of such architecture using formalisms like goal-driven BDI architectures, Pearl's Do calculus and Description Logics. Semantic web technology is used extensively in the adoption of such formalisms.
Up to this point, the agent architecture supports distributed problem solving and doesn't make evident the participation of human users. In order to do so, we will use our agent architecture in the context of Electronic Institution[8]. Agent classes will be used for elaborating plans and determining which roles an agent can play on institutional protocols or scenes. Dynamic instantiation of agents in conjunction with a Directory Facilitator will be used for optimizing resources allocation through the selection of the best agent (existing or not) for a given scene.

\section{ACKNOWLEDGMENT}

The authors would like to thank to the anonymous reviewers of this work and to professor Pablo Noriega for their valuable comments.

\section{REFERENCES}

[1] Tomas Alvira, Luis Clavell, and Tomas Melendo. Metafisica. EUNSA, 2001.

[2] F. Baader, C. Lutz, M. Milicic, U. Sattler, and F. Wolter. Integrating description logics and action formalisms: First results. In AAAI, editor, Proceedings of the Twentieth National Conference on Artificial Intelligence (AAAI-05), 2005.

[3] Franz Baader. The Description Logic Handbook: Theory, Implementation, and Applications. Cambridge University Press, September 2007.

[4] Fabio L. Bellifemine, Giovanni Caire, and Dominic Greenwood. Developing Multi-Agent Systems with JADE (Wiley Series in Agent Technology). Wiley, April 2007.

[5] Eva Bou, Maite Lopez-sanchez, and J. A. Rodriguez-aguilar. Towards self-configuration in autonomic electronic institutions. In In: COIN 2006 Workshops. Number LNAI 4386, pages 220-235. Springer, 2007.

[6] Hector Ceballos and Francisco Cantu. Integrating semantic annotations in bayesian causal models. In 20th International Workshop on Description Logics (DL07), pages 527-528, June 2007.

[7] P.R. Cohen and Hector J. Levesque. Intention is choice with commitment. Artificial Intelligence, (42):213, 1990.

[8] Marc Esteva, Juan A. Rodriguez-Aguilar, Josep L1. Arcos, Carles Sierra, and Pere Garcia. Formalising agent mediated electronic institutions. In Agent Mediated Electronic Commerce, LNAI 1991, pages 29-38. Springer-Verlag, 2000.

[9] Mike Georgeff, Barney Pell, Martha Pollack, Milind Tambe, and Mike Wooldridge. The belief-desire-intention model of agency. In Jörg Müller, Munindar P. Singh, and Anand S. Rao, editors, Proceedings of the 5th International Workshop on Intelligent Agents V:Agent Theories, Architectures, and Languages (ATAL-98), volume 1555, pages 1-10. Springer-Verlag: Heidelberg, Germany, 1999.

[10] Enrico Giunchiglia, Joohyung Lee, Vladimir Lifschitz, Norman McCain, Hudson Turner, and Joohyung Lee Vladimir Lifschitz. Nonmonotonic causal theories. Artificial Intelligence, 153:2004, 2004.

[11] Ian Horrocks, Sergio Tessaris, Ulrike Sattler, and Stephan Tobies. How to decide query containment under constraints using a description logic. In In Proc. of LPAR2000, pages 326-343. Springer, 1999.

[12] Allen Newell. Unified Theories of Cognition. Harvard University Press, 1994.

[13] Judea Pearl. Causality. Models, Reasoning, and Inference. Cambridge University Press, 2000.

[14] Alexander Pokahr, Lars Braubach, and Winfried Lamersdorf. EXP In Search of Innovation (Special Issue on JADE), volume 3, chapter Jadex: Implementing a BDI-Infrastructure for JADE Agents, pages 7685. Telecom Italia Lab, 2003.

[15] David Poole. The independent choice logic for modelling multiple agents under uncertainty. Artificial Intelligence, 1-2(94):7-56, 1997.

[16] Munindahr P. Singh. Commitments among autonomous agents in information-rich environments. In Modelling Autonomous Agents in a Multi-Agent World, pages 141-155, 1997.

[17] Michael Wooldridge. Reasoning about Rational Agents. MIT, 2000. 